\section{DIESEL PARTICULATES IN ALLERGIC AIRWAY DISEASE}

Andrew Saxon, M.D.

Professor of Medicine, Chief Division of

Clinical Immunology/Allergy,

Department of Medicine, UCLA School of

Medicine,

Los Angeles, CA

Particulates from fossil fuel combustion are common and linked to allergic airway disease. Changes in the quality of the air we breathe related to air borne "pollutants" are likely one critical factor in the increasing worldwide incidence, prevalence, and severity of allergic airway disease. The dramatic rise in expression of human allergic airway disease in the past two hundred years parallels the increase in fossil fuel combustion - - airway allergic disease was even called a disease of industrialization as early as 1907. Burning of fossil fuels generates a variety of xenobiotic compounds among which the polyaromatic hydrocarbons (PAH) and monofunctional inducers of oxidative stress (MIOS) are of particular interest. This type of pollution has increased 1000 's of fold since the industrial revolution began. The enormity and extent of particulates generated by anthropomorphic activities can be appreciated by the evidence that a global "particulate haze" may now be reflecting enough sunlight to induce global cooling and partially counter effects of global warming .

Diesel exhaust particles (DEP) are of interest because their occurrence has been reasonably well studied both over time and quantitatively under a variety of circumstances. Thus they can serve as a model for airborne particulates containing PAH/MIOS. DEP make up, on average, $40 \%$ of the 10 micron particles (PM10) in a city such as Los Angeles. DEP are representational of PAH/MIOS containing particulates generated from a variety of human activities, e.g. burning of fossil fuels and cigarette smoking. Because of their wide spread nature, such particulates are universally found in indoors and often in concentrations equal to or exceeding that found in ambient outdoor air. Furthermore, non-occupational settings (i.e. sitting at a busy bus stop in L.A.) can result in acute exposures as high as 100 times the recommended peak concentrations.
Epidemiological studies have linked increased incidence of allergic respiratory disease to airborne pollution. The arguments in favor and against the role of pollutants in the clinical expression of allergic airways disease has been debated in many articles. There is no simple answer - the final expression of clinical illness is very complex. Our studies on DEP focus on a somewhat different issue: whether the presence of DEP, and similar organic respirable materials containing PAH/MIOS, act as mucosal adjuvants to help initiate or enhance IgE production to common inhaled allergens. We are particularly interested in the role polycyclic aromatic hydrocarbons play in increasing the incidence of allergic sensitization and thereby allergic airway disease. This is not to say that acute or chronic exposure to airborne pollutants do not directly exacerbate allergic airway disease by non-specific mucosal inflammatory effects. Our investigation is directed at the more subtle but, we feel more far reaching issue, of the role of airborne PAH in inducing or enhancing the specific IgE response in humans. Increased allergenspecific IgE production is the substrate for development of allergic airway disease.

The chemicals contained in combustion particulates induce important metabolic pathways. Combustion generated PAH and MIOS mediate their effects via a pair of interdigitating metabolic pathways Briefly, PAH (bifunctional inducers) directly enter cells and bind to the cytosolic Ah receptor complex. This complex is transported to the nucleus where it binds to xenobiotic response elements (XRE) and induces a number of genes (phase I induction). Key among these is the P450 CYP1A1 gene which in turn metabolizes the PAH to MIOSs. MIOSs produced by $\mathrm{PAH}$ metabolism and those occurring in combustion products e.g. quinones are monofunctional inducers which act to alter the redox state of the cell and induce the anti-oxidant response element (ARE) in a number of phase II genes, (e.g. NQO1) and possibly cytokine and chemokine genes. These metabolic pathways provide opportunities to study their specific induction by measuring CYP1A1 or NQO1 and thereby determine their involvement in various effects observed. Furthermore, in the in vitro and to a lesser extent, in the in vivo studies, one can use compounds that specifically induce or block phase I or phase II effects to dissect the 
mechanism by which DEP/PAH effects are mediated.

Animals studies demonstrate DEP and PAH enhancement of IgE antibodies. Miyamoto and colleagues performed the initial in vitro and in vivo studies with mice demonstrating a direct effect of diesel exhaust particles on total and antigen specific IgE antibody production. Intraperitoneal and intranasal inoculation of DEP increased the IgE response to both ovalbumin and Japanese cedar pollen. Subsequently a host of animal studies have shown that DEP can result in a) an increase in eosinophilic inflammation b) an shift toward increased $\mathrm{TH}_{2}$ cytokines including an increase in IL-4 production in the bronchial lymph nodes, c) increased bronchial reactivity following intratracheal instillation of DEP. Furthermore a number of these effects can be elicited by benzo(a)pyrene, a PAH constituent of DEP. These animal studies support the hypothesis that PAH/MIOS can lead both to 1) induction of antigen specific IgE responses under conditions where antigen alone would not induce IgE antibody and 2) enhancement of ongoing IgE responses.

Human IgE responses in relationship to DEP and PAH/MIOS. We have employed human systems to investigate the effects of DEP and the chemical contained therein upon allergic inflammation. We initially demonstrated that DEP induced enhanced IgE production both in vitro and in vivo. Combined allergen DEP challenge led to a marked increase in local allergen specific IgE production. DEP was shown to markedly enhance mucosal TH0-like cytokine production at 24 hours. However, the presence of allergen, this cytokine production shows TH2-like deviation associated with increased IgE antibody production. However, the signal(s) driving the observed TH2-like deviation remains unknown, both as to its cell source and molecular nature. DEP have recently been shown to directly bind allergens molecules and thereby provide a mechanism for "natural co-administration".

Investigation into the initial cellular response has shown that there is a burst of chemokine production from non-T cells within hours of in vivo DEP exposure. Maximal DEP-enhanced expression was at 2 hours for RANTES and MCP-3 and at 4 to 6 hours for MIP-1a and all had diminished by 24 hours. In contrast, DEP did not enhance expression of eotaxin mRNA at any time demonstrating that the action of DEP was not simply a global effect on all CC chemokines.
Challenge with DEP also resulted in an increase in total and mononuclear cell counts in nasal lavage fluids and a significant enhancement of ECP levels at 6 to 24 hours after challenge. We undertook to provide evidence for local DEP driven in vivo isotype switching to IgE following nasal challenges. Detection of deleted switch circular DNA (switch circles) was employed as definitive molecular evidence of $\mathrm{Ig}$ isotype switching. Four days after combined intra-nasal DEP plus ragweed challenge, we detected and characterized clones of deleted switch circular DNA $\left(\mathrm{S}_{\varepsilon} / \mathrm{S} \mu\right)$ representing switching from $\mu$ to $\varepsilon$ in mucosal $B$ cells. No switch circular DNA was detected in nasal lavage cells following challenge with DEP alone nor with ragweed allergen alone. These results indicate that the combination of mucosal stimulation with DEP and ragweed allergen is capable of driving in vivo isotype switching to IgE in humans with ragweed allergy. We are presently trying to determine if DEP, in combination with a neo-antigen, can induce primary sensitization.

A key question in these studies is what are the molecular pathways by which PAH and their conversion products drive allergic inflammation. Circumstantial evidence suggested that macrophages are involved. Thus we demonstrated that a representative PAH, $\quad \beta$-napthoflavone (BNF), and a representative quinone metabolite, tertbutylhydroxyquinone (tBHQ), induce Jun kinase (JNK) and p38MAPK activities in parallel with the generation of AP-1 mobility shift complexes in human macrophage cell lines. Activation of MAP kinases was dependent on generation of oxidative stress and was inhibited by $\mathrm{N}$-acetylcysteine. Another pathway linked to $\mathrm{PAH}$ is the antioxidant response element (ARE), which regulates expression of detoxifying enzymes. BNF and tBHQ both activated a human ARE reporter gene in macrophages. While the ARE core contains a consensus AP-1 sequence, AP-1 was not required for hARE activation, suggesting that $P A H$ and their conversion products operate via ARE-specific transcription factors. These data establish important activation pathways for PAH in the human immune system and provide potential molecular targets to modulate the effect of environmental pollutants on allergic inflammation. 